\title{
A precise determination of the HVP contribution to the muon anomalous magnetic moment from lattice QCD
}

\author{
Christoph Lehner ${ }^{1, \star}$ for the RBC and UKQCD collaborations \\ ${ }^{1}$ Physics Department, Brookhaven National Laboratory, Upton, NY 11973, USA
}

\begin{abstract}
In this talk I present the current status of a precise first-principles calculation of the quark connected, quark disconnected, and leading QED and strong isospin-breaking contributions to the leading-order hadronic vacuum polarization by the RBC and UKQCD collaborations. The lattice data is also combined with experimental $e^{+} e^{-}$scattering data, consistency between the two datasets is checked, and a combined result with smaller error than the lattice data and $e^{+} e^{-}$scattering data individually is presented.
\end{abstract}

\section{Introduction}

The anomalous magnetic moment

$$
a_{\ell}=\left(g_{\ell}-2\right) / 2
$$

of a particle $\ell$ encodes the radiative corrections to Dirac's result $g_{\ell}=2$. These moments are experimentally measured to great precision for the light leptons $e$ and $\mu$ and therefore provide a stringent test of the standard model (SM) of particle physics. Since contributions of new particles beyond the SM are in general proportional to the square of the lepton's mass one may expect the anomalous magnetic moment of the muon to be a particularly interesting target to find new physics. Intriguingly, there is a long-standing tension at the $3 \sigma-4 \sigma$ level between the SM theory prediction and the BNL E821 experimental result, see Tab. 1.

With the anticipated fourfold reduction in experimental uncertainty targeted by the Fermilab E989 experiment, a similar reduction in theory uncertainty is needed. In this work, we address how to reduce the uncertainty in the SM prediction of the leading-order (LO) hadronic vacuum polarization (HVP) contribution to $a_{\mu}$.

\section{Lattice methodology}

In order to match the target precision of the Fermilab E989 experiment, see Tab. 1, we need to compute quark-connected, quark-disconnected, and QED and isospin-breaking contributions to the HVP. The corresponding diagrams in terms of quark and photon fields are depicted in Fig. 1. The quarkconnected piece, and in particular the contribution of up and down quarks, is the largest individual component and needs to be computed at the sub-percent level. The other contributions are suppressed by at least a factor of ten and therefore have reduced precision requirements.

\footnotetext{
^Speaker, e-mail: clehner@bnl.gov
} 


\begin{tabular}{lrr}
\hline \hline Contribution & Value $\times 10^{10}$ & Uncertainty $\times 10^{10}$ \\
\hline QED (5 loops) & 11658471.895 & 0.008 \\
EW & 15.4 & 0.1 \\
HVP LO & 692.3 & 4.2 \\
HVP NLO & -9.84 & 0.06 \\
HVP NNLO & 1.24 & 0.01 \\
Hadronic light-by-light & 10.5 & 2.6 \\
\hline Total SM prediction & 11659181.5 & 4.9 \\
\hline BNL E821 result & 11659209.1 & 6.3 \\
FNAL E989/J-PARC E34 goal & & $\approx 1.6$ \\
\hline \hline
\end{tabular}

Table 1. Overview of individual contributions and uncertainties to $a_{\mu}$.
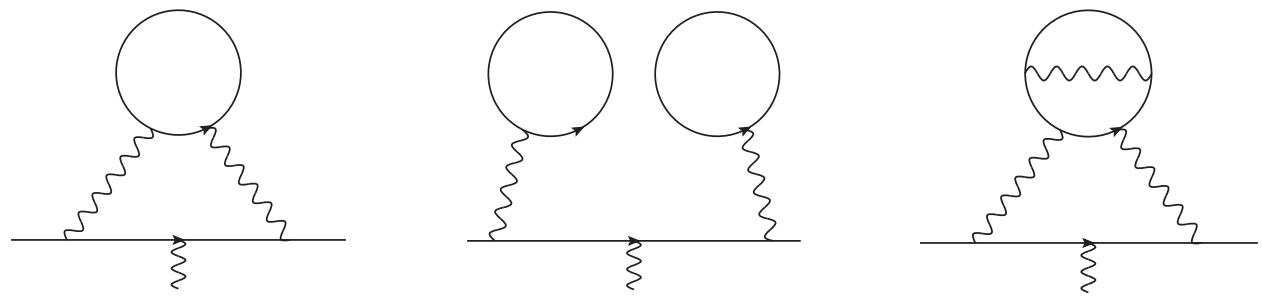

Figure 1. Quark-connected (left), quark-disconnected (center), and a QED-correction (right) diagram.

In the following sections, we present first-principles lattice QCD results for all relevant contributions measured on RBC and UKQCD domain-wall ensembles at effectively physical pion mass. We use the $48^{3}$ and $64^{3}$ ensembles whose properties are described in detail in Ref. [1]. We would like to highlight that due to the precise knowledge of the $\Omega^{-}$mass in both experiment and on the lattice, the lattice spacing is known to 2-3 parts per thousand on these ensembles. QED and isospin breaking corrections to this mass are small and will be presented in an upcoming publication, see Ref. [2]. As was observed recently in Ref. [3] such high precision on the lattice spacing is important for a high-precision calculation of $a_{\mu}$.

It is convenient to discuss the lattice data in the representation of Ref. [4] and write

$$
a_{\mu}^{\mathrm{HVP}}=\sum_{t=0}^{\infty} w_{t} C(t)
$$

with

$$
C(t)=\frac{1}{3} \sum_{\vec{x}} \sum_{j=0,1,2}\left\langle J_{j}(\vec{x}, t) J_{j}(0)\right\rangle
$$

where

$$
J_{\mu}(x)=i \sum_{f} Q_{f} \bar{\Psi}_{f}(x) \gamma_{\mu} \Psi_{f}(x)
$$


is the vector current with sum over all quark flavors $f$ with QED charge $Q_{f}$. The coefficient $w_{t}$ captures the photon and muon part of the diagram and can be obtained in a continuum calculation as proposed in [5]. Throughout our lattice calculation we use appropriately normalized local vector currents.

\subsection{Quark-connected contribution}

There are two significant obstacles to a high-precision calculation of the quark-connected contribution to $a_{\mu}$ : the statistical precision deteriorates exponentially at long distances and the finite-volume errors may be non-negligible [6]. In particular the light-quark contribution suffers from these uncertainties. In Fig. 2 we show the weighted correlator $w_{t} C(t)$ and a corresponding scalar QED calculation in position space that we will subsequently use to correct leading finite-volume effects. The precision degradation at long distances is clearly visible.

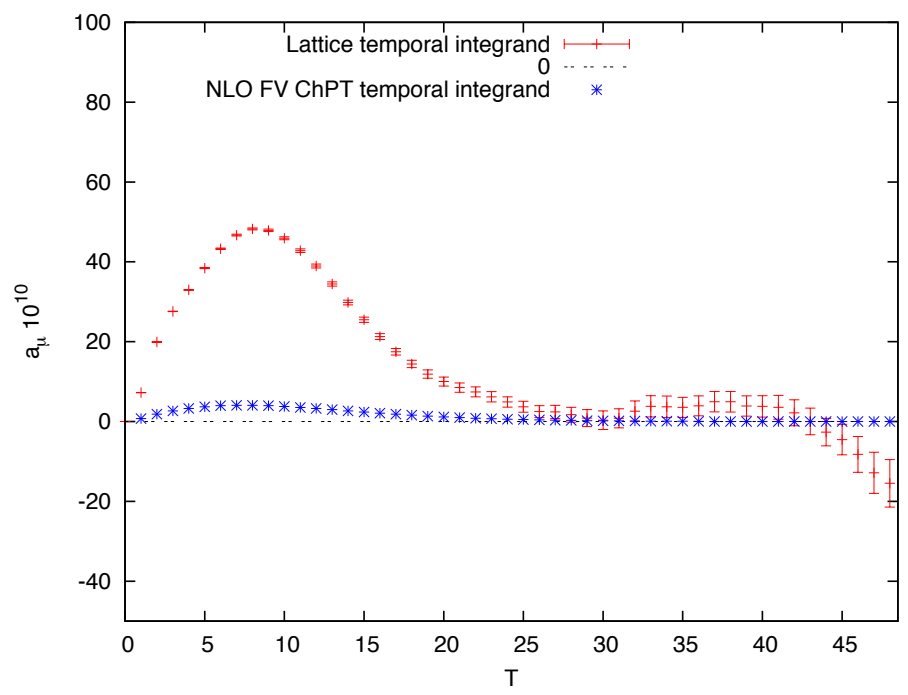

Figure 2. Integrand $w_{T} C(T)$ for the light-quark connected contribution with $m_{\pi}=140 \mathrm{MeV}$ and $a=0.11 \mathrm{fm}$.

We employ a multi-step approximation scheme with low-mode averaging [7] over the entire volume and two levels of approximations in a stopped deflated solver (AMA) [8-11] of randomly positioned point sources. Figure 3 contrasts the statistical precision of this improved estimator with the traditional choice of $Z_{2}$ wall sources and we observe a reduction of noise by one order of magnitude at same cost. We use low modes obtained through a new Lanczos method working on multiple grids [12], which yields an approximate tenfold reduction in memory cost for our ensembles at physical pion mass with approximately $5 \mathrm{fm}$ box size.

For the strange-quark quark-connected contribution we use the dataset published in Ref. [13].

\subsection{Quark-disconnected contribution}

Our result for the quark-disconnected diagram at physical pion mass is published in Ref. [14], where we find

$$
a_{\mu}^{\mathrm{HVP}(\mathrm{LO}) \mathrm{DISC}}=-9.6(3.3)_{\text {stat }}(2.3)_{\text {sys }} \times 10^{-10} .
$$



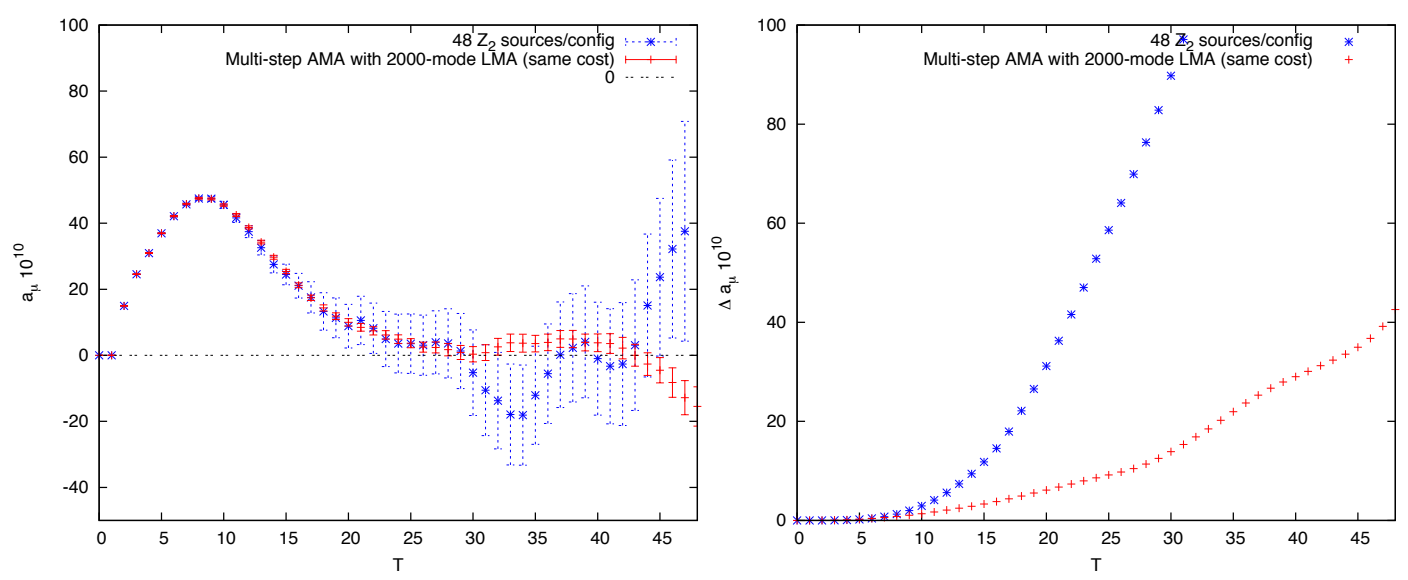

Figure 3. Significant error reduction using full-volume low-mode average in addition to a multi-level all-mode average.

\subsection{QED and strong isospin-breaking corrections}

We compute both QED corrections and strong isospin-breaking corrections to our result, see Figs. 4 and 5, respectively. We utilize an importance sampling technique in position space similar to Ref. [15] and an appropriately normalized local vector current coupling to the photon fields. The photons are regulated with the $\mathrm{QED}_{L}$ prescription in this work and the universal $1 / L$ and $1 / L^{2}$ corrections are taken into account for the mass spectrum [16]. The use of an infinite-volume photon to reduce finite-volume effects in the QED corrections to the HVP is currently also being explored and will be presented in Ref. [2]. The results presented in this section are at physical pion mass improving over our recent work [17].

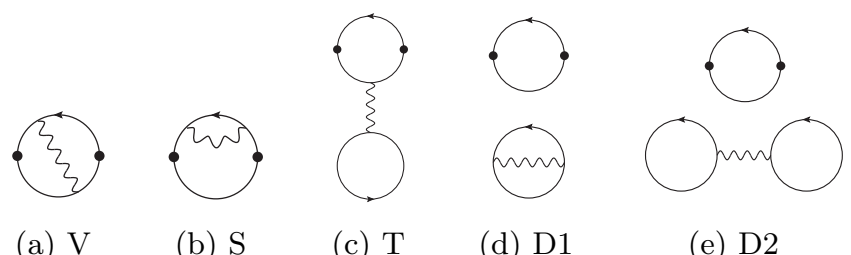
(a) $\mathrm{V}$
(b) $\mathrm{S}$
(c) $\mathrm{T}$
(d) D1
(e) D2

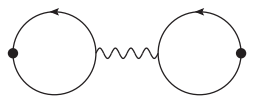

(f) $\mathrm{F}$

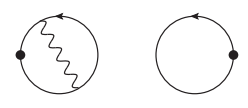

(g) D3

Figure 4. QED correction diagrams.

We first compute QED and strong isospin-breaking corrections to the $K^{0}, K^{+}, \pi^{0}, \pi^{+}$, and $\Omega^{-}$ masses and re-tune the up, down, and strange quark mass as well as the lattice spacing by matching these masses to their experimental values. 


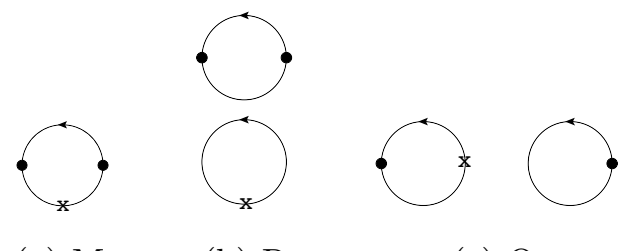
(a) M
(b) $\mathrm{R}$
(c) $\mathrm{O}$

Figure 5. Strong isospin-breaking correction diagrams. The crosses denote a sequential insertion of a scalar operator.

The re-tuned quark masses and the lattice spacing can be expanded around the values of the pure QCD simulation with light quark mass $m_{l}$, strange quark mass $m_{h}$, and lattice spacing $a^{(0)}$. We write

$$
\begin{aligned}
m_{u} & =m_{l}+\Delta m_{u}, \\
m_{d} & =m_{l}+\Delta m_{d}, \\
m_{s} & =m_{h}+\Delta m_{s}, \\
a & =a^{(0)}+a^{(1)} .
\end{aligned}
$$

The pion and kaon spectrum provides the following conditions

$$
\begin{aligned}
& a m_{\pi^{+}}^{(\text {exp })}=m_{\pi}^{(0)}+m_{\pi^{+}}^{(1, \mathrm{QED})}+m_{\pi^{+}}^{(1, u)} \Delta m_{u}+m_{\pi^{+}}^{(1, d)} \Delta m_{d}, \\
& a m_{\pi^{0}}^{(\text {exp })}=m_{\pi}^{(0)}+m_{\pi^{0}}^{(1, \mathrm{QED})}+m_{\pi^{0}}^{(1, u)} \Delta m_{u}+m_{\pi^{0}}^{(1, d)} \Delta m_{d}, \\
& a m_{K^{+}}^{(\text {exp })}=m_{K}^{(0)}+m_{K^{+}}^{(1, \mathrm{QED})}+m_{K^{+}}^{(1, u)} \Delta m_{u}+m_{K^{+}}^{(1, s)} \Delta m_{s}, \\
& a m_{K^{0}}^{(\text {exp })}=m_{K}^{(0)}+m_{K^{0}}^{(1, \mathrm{QED})}+m_{K^{0}}^{(1, d)} \Delta m_{d}+m_{K^{0}}^{(1, s)} \Delta m_{s},
\end{aligned}
$$

where only leading QED $\left(m^{(1, \mathrm{QED})}\right)$ and mass-correction $\left(m^{(1, u / d / s)}\right)$ effects have been included. The effect of the lattice-spacing shift can be made more explicit in

$$
\begin{aligned}
& a^{(0)} m_{\pi^{+}}^{(e x p)}=m_{\pi}^{(0)}+m_{\pi^{+}}^{(1, \mathrm{QED})}+m_{\pi^{+}}^{(1, u)} \Delta m_{u}+m_{\pi^{+}}^{(1, d)} \Delta m_{d}-a^{(1)} m_{\pi}^{(\exp )}, \\
& a^{(0)} m_{\pi^{0}}^{(e x p)}=m_{\pi}^{(0)}+m_{\pi^{0}}^{(1, \mathrm{QED})}+m_{\pi^{0}}^{(1, u)} \Delta m_{u}+m_{\pi^{0}}^{(1, d)} \Delta m_{d}-a^{(1)} m_{\pi}^{(\exp )}, \\
& a^{(0)} m_{K^{+}}^{(e x p)}=m_{K}^{(0)}+m_{K^{+}}^{(1, \mathrm{QED})}+m_{K^{+}}^{(1, u)} \Delta m_{u}+m_{K^{+}}^{(1, s)} \Delta m_{s}-a^{(1)} m_{K}^{(e x p)}, \\
& a^{(0)} m_{K^{0}}^{(e x p)}=m_{K}^{(0)}+m_{K^{0}}^{(1, \mathrm{QED})}+m_{K^{0}}^{(1, d)} \Delta m_{d}+m_{K^{0}}^{(1, s)} \Delta m_{s}-a^{(1)} m_{K}^{(e x p)} .
\end{aligned}
$$

If we restrict the discussion of mass-shifts to diagram $M$, a further simplification can be obtained

$$
\begin{aligned}
& a^{(0)} m_{\pi^{+}}^{(\text {exp })}=m_{\pi}^{(0)}+m_{\pi^{+}}^{(1, \mathrm{QED})}+m_{\pi^{+}}^{(1, u)}\left(\Delta m_{u}+\Delta m_{d}\right)-a^{(1)} m_{\pi}^{(\text {exp })}, \\
& a^{(0)} m_{\pi^{0}}^{(\text {exp })}=m_{\pi}^{(0)}+m_{\pi^{0}}^{(1, \mathrm{QED})}+m_{\pi^{+}}^{(1, u)}\left(\Delta m_{u}+\Delta m_{d}\right)-a^{(1)} m_{\pi}^{(\text {exp })}, \\
& a^{(0)} m_{K^{+}}^{(\text {exp })}=m_{K}^{(0)}+m_{K^{+}}^{(1, \mathrm{QED})}+m_{K^{+}}^{(1, u)} \Delta m_{u}+m_{K^{+}}^{(1, s)} \Delta m_{s}-a^{(1)} m_{K}^{(\text {exp })}, \\
& a^{(0)} m_{K^{0}}^{(\text {exp })}=m_{K}^{(0)}+m_{K^{0}}^{(1, \mathrm{QED})}+m_{K^{0}}^{(1, d)} \Delta m_{d}+m_{K^{0}}^{(1, s)} \Delta m_{s}-a^{(1)} m_{K}^{(\text {exp })},
\end{aligned}
$$

which makes it apparent that $a^{(1)}$ and $\Delta m_{u}+\Delta m_{d}$ cannot be independently obtained from the above set of equations. The expected smaller size of diagram $\mathrm{O}$ compared to diagram $\mathrm{M}$ likely makes it at 
least numerically challenging to obtain a precise determination of $a^{(1)}$ as well as the mass shifts from the above set of equations. We are therefore encouraged to use an alternative mass scale to determine $a^{(1)}$ for which we will use the mass of the $\Omega^{-}$.

If we ignore the lattice-spacing shift for now and only focus on diagrams $V, S$, and $M$, we obtain

$$
\begin{aligned}
\Delta m_{u} & =-0.000678(83), \\
\Delta m_{d} & =0.000519(83), \\
\Delta m_{s} & =-0.000431(32) .
\end{aligned}
$$

The re-tuning of the lattice spacing is a small effect that is absent in this work, however, will be presented separately in Ref. [2].

Figure 6 displays the pion mass splitting taking into account the finite-volume corrections and shows agreement with the experimental measurement.

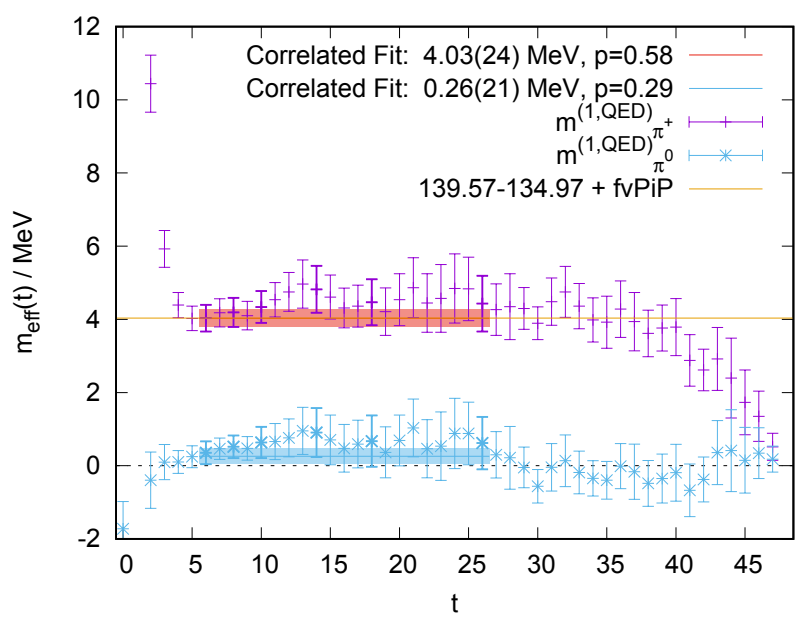

Figure 6. Pion mass splitting at physical pion mass from the RBC/UKQCD $48^{3}$ ensemble. The lattice data shown here is not yet finite-volume corrected, however, to facilitate a proper comparison we also show the result of adding the universal $\mathrm{QED}_{L}$ finite-volume correction (fvPiP) to the experimental pion mass splitting.

We are now in a position to compute the QED and strong isospin-breaking corrections to $a_{\mu}^{\mathrm{HVP}} \mathrm{LO}$. In this work, results for the electro-quenched diagrams $S, V$, and $F$ are shown. In addition to the absence of a re-tuning of the lattice spacing, also the effects of QED corrections on the local-current renormalization are ignored in this work. These effects are small and will be presented elsewhere [2].

The valence strong isospin-breaking effect (diagram $\mathrm{M}$ ) is shown in Fig. 7 and the diagrams $\mathrm{S}$ and $\mathrm{V}$ are shown in Fig. 8.

\section{A combined analysis of lattice data and R-ratio data}

The lattice data presented so far is sufficient to attempt a comparison to the $e^{+} e^{-}$scattering data. We connect $C(t)$ to the R-ratio data [4] writing

$$
\Pi\left(-Q^{2}\right)=\frac{1}{\pi} \int_{0}^{\infty} d s \frac{s}{s+Q^{2}} \sigma\left(s, e^{+} e^{-} \rightarrow \mathrm{had}\right)
$$




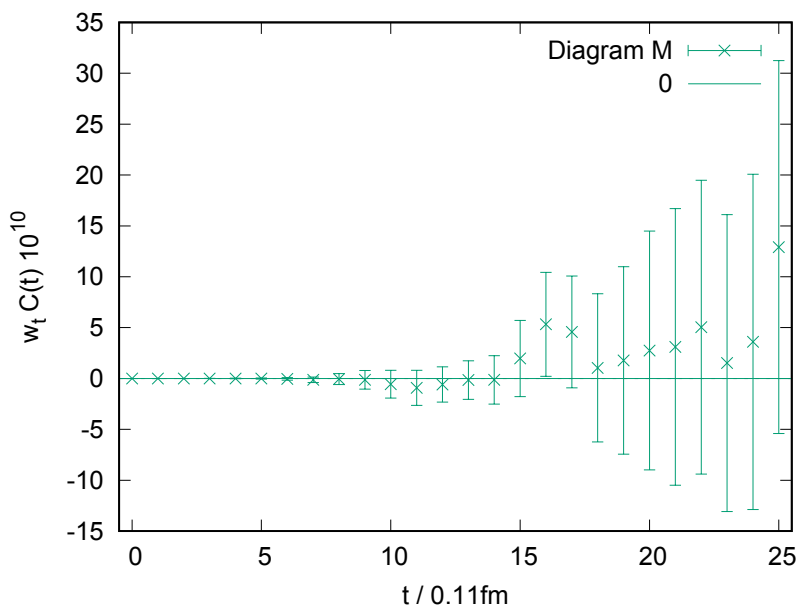

Figure 7. Valence strong isospin-breaking correction to $a_{\mu}^{\mathrm{HVP} \text { LO }}$.

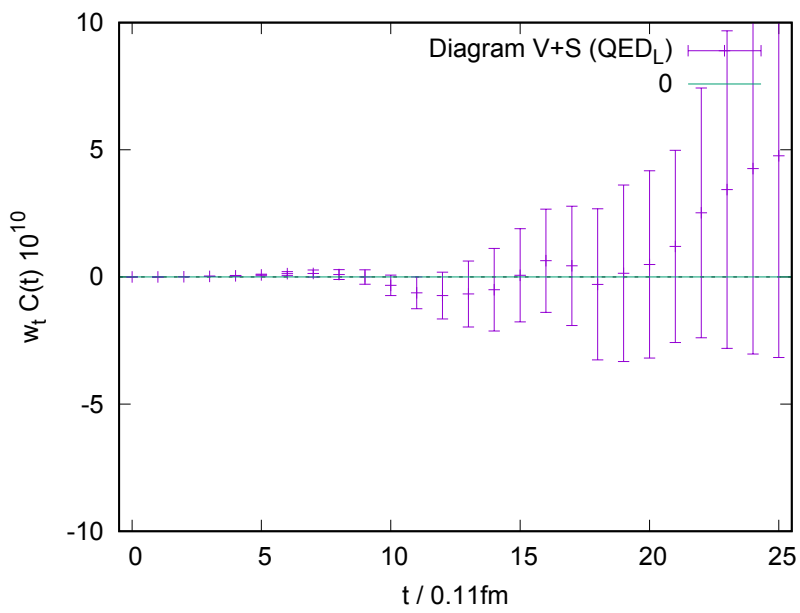

Figure 8. QED corrections from diagrams $\mathrm{S}$ and $\mathrm{V}$ to $a_{\mu}^{\mathrm{HVP} \mathrm{LO}}$.

with

$$
R(s)=\frac{\sigma\left(s, e^{+} e^{-} \rightarrow \text { had }\right)}{\sigma\left(s, e^{+} e^{-} \rightarrow \mu^{+} \mu^{-}, \text {tree }\right)}=\frac{3 s}{4 \pi \alpha^{2}} \sigma\left(s, e^{+} e^{-} \rightarrow \text { had }\right) .
$$

A Fourier transform then gives

$$
C(t) \propto \int_{0}^{\infty} d(\sqrt{s}) R(s) s e^{-\sqrt{s} t} \equiv \int_{0}^{\infty} d(\sqrt{s}) \rho(\sqrt{s}) e^{-\sqrt{s} t}
$$

which defines a density function $\rho(\sqrt{s})$. In the remainder of this presentation, we take $R(s)$ from Ref. [18] and for now treat values for different $s$ to be fully correlated. This imperfection is removed in Ref. [2]. Figure 9 shows the spectral density function in the convention of Eq. (27). 


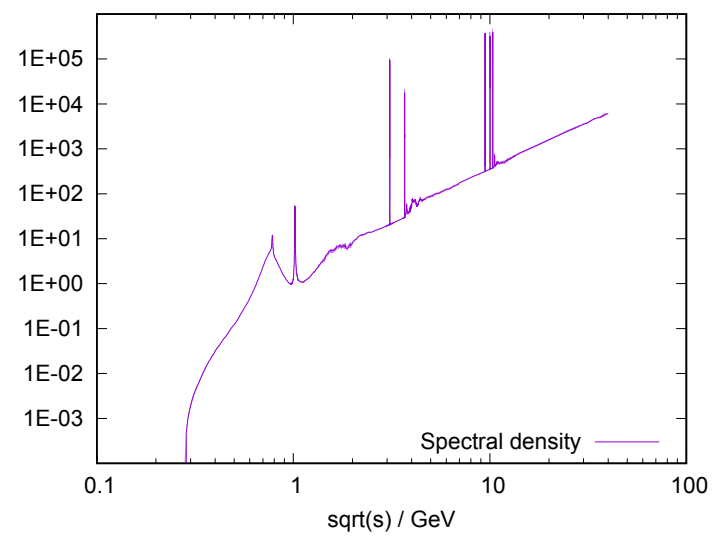

Figure 9. Spectral density function in the convention of Eq. (27).

In Fig. 10 we contrast $a_{\mu}$ in the representation of Eq. (2) from the R-ratio data with lattice data in the isospin symmetric limit at two different lattice spacings. We observe reasonable agreement and notice that while the lattice data is most precise at short distances, the R-ratio data behaves in a complementary manner.

\subsection{Window method}

We define a window method similar to Ref. [4] with the intention to combine the regions of respective high precision from the R-ratio and lattice data sets. We select a window in $t$ by defining a smeared $\Theta$ function

$$
\Theta(t, \mu, \sigma) \equiv[1+\tanh [(t-\mu) / \sigma]] / 2
$$

which we find to be helpful to control the effect of discretization errors by the smearing parameter $\sigma$.
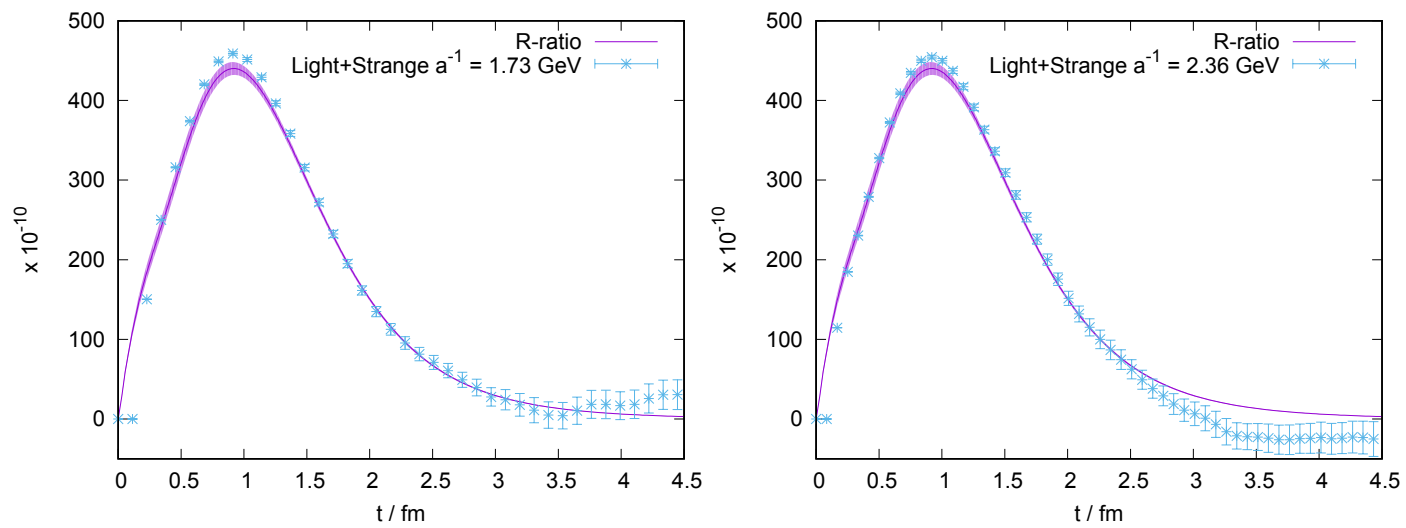

Figure 10. Comparison of R-ratio and lattice data. 

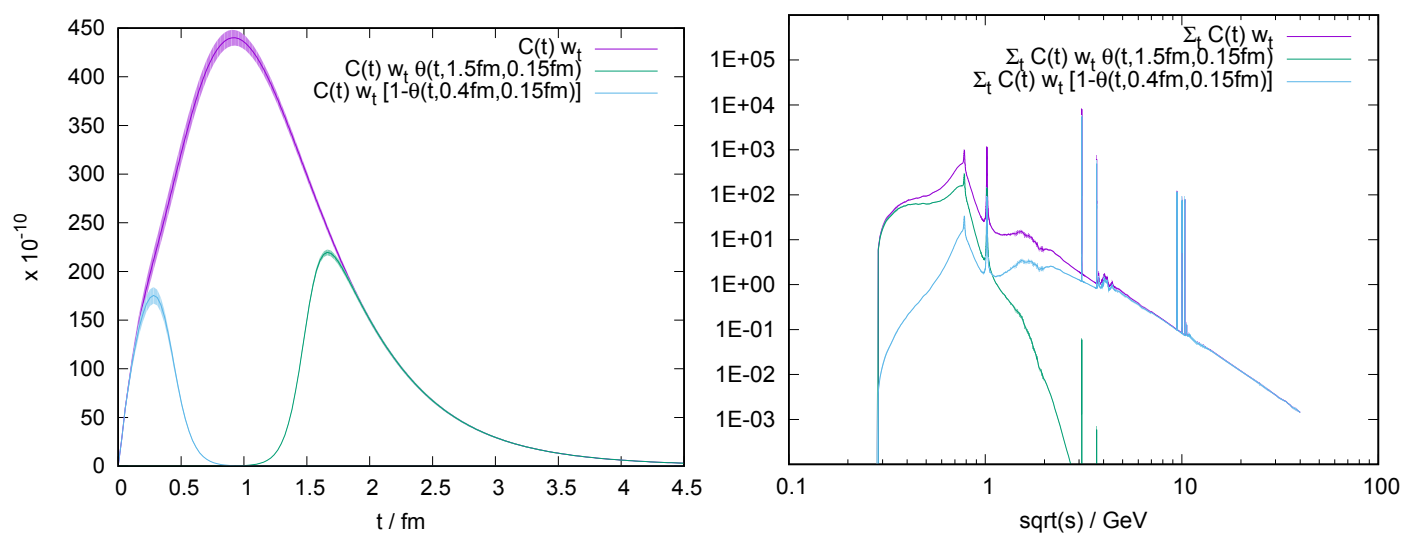

Figure 11. Window of R-ratio data in Euclidean position space (left) and the effect of the window in terms of re-weighting energy regions (right).

We define the "window method" as

$$
a_{\mu}=\sum_{t} w_{t} C(t) \equiv a_{\mu}^{\mathrm{SD}}+a_{\mu}^{\mathrm{W}}+a_{\mu}^{\mathrm{LD}}
$$

with

$$
\begin{aligned}
& a_{\mu}^{\mathrm{SD}}=\sum_{t} C(t) w_{t}\left[1-\Theta\left(t, t_{0}, \Delta\right)\right] \\
& a_{\mu}^{\mathrm{W}}=\sum_{t} C(t) w_{t}\left[\Theta\left(t, t_{0}, \Delta\right)-\Theta\left(t, t_{1}, \Delta\right)\right], \\
& a_{\mu}^{\mathrm{LD}}=\sum_{t} C(t) w_{t} \Theta\left(t, t_{1}, \Delta\right)
\end{aligned}
$$

where each contribution is accessible from both lattice and R-ratio data.

We then take both the short-distance and long-distance region from the R-ratio data aided by perturbative QCD [18]. The removal of the short-distance region reduces discretization errors in our calculation, however, is not strictly required to achieve high precision.

In Fig. 11 we show the effect of the window selection in Euclidean position space and the corresponding effect in terms of re-weighting different energy regions in their contribution to $a_{\mu}$. The suppression of short distances has a pronounced effect of high-energy contributions as expected. In addition the contribution for long Euclidean distances comes mostly from the lower end of the energy spectrum.

Finally Fig. 12 contrasts lattice and R-ratio data for the window with $t_{0}=0.4 \mathrm{fm}, t_{1}=1.5 \mathrm{fm}$, and $\Delta=0.15 \mathrm{fm}$. We observe good agreement between both datasets.

\subsection{Continuum limit}

The continuum limit of our domain-wall fermion lattice calculations at physical pion mass is mild. This is illustrated in Fig. 13 for the light-quark contribution to $a_{\mu}^{\mathrm{W}}$ with $t_{0}=0.4 \mathrm{fm}$ and $\Delta=0.15$ 


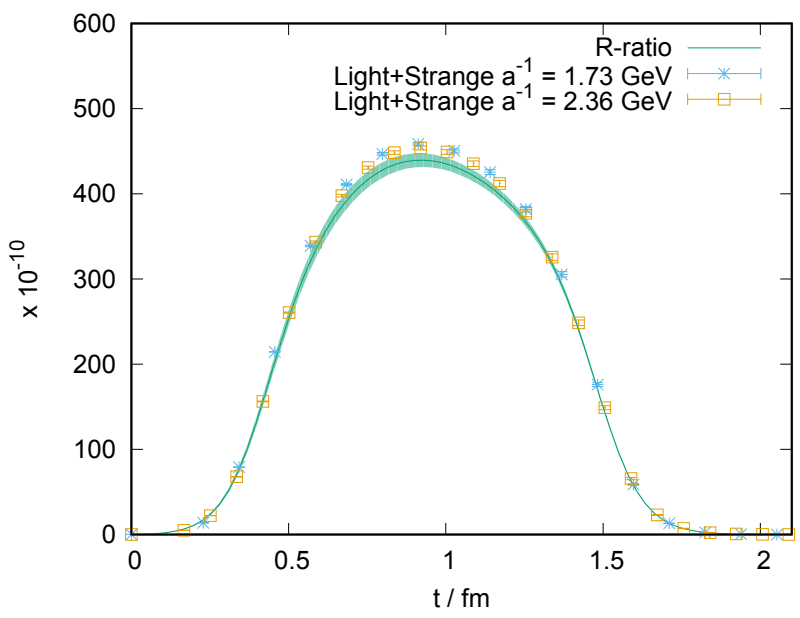

Figure 12. Example contribution to $a_{\mu}^{\mathrm{W}}$ with $t_{0}=0.4 \mathrm{fm}, t_{1}=1.5 \mathrm{fm}, \Delta=0.15 \mathrm{fm}$.

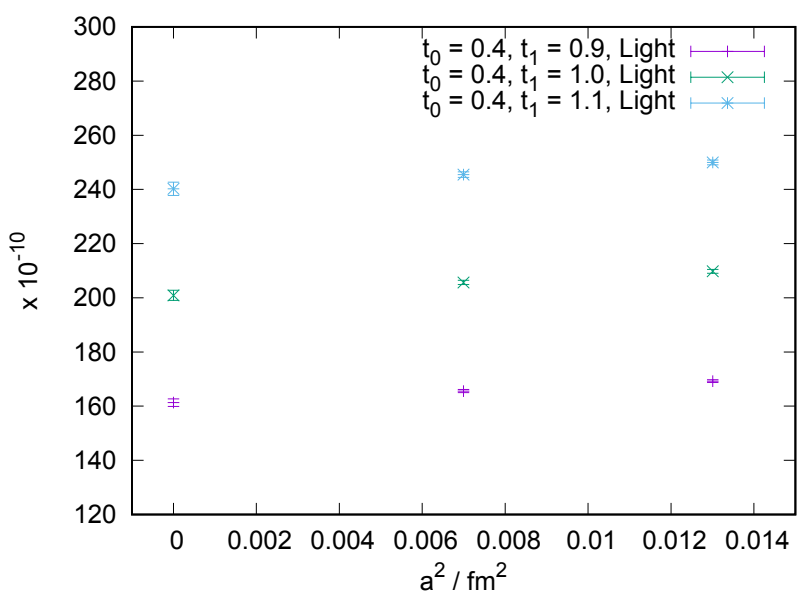

Figure 13. Continuum limit of $a_{\mu}^{\mathrm{W}}$ with $t_{0}=0.4 \mathrm{fm}$ and $\Delta=0.15 \mathrm{fm}$.

fm, where our coarse-lattice result only differs from the continuum extrapolation at the level of a few per-cent. This is in contrast to the rather steep continuum extrapolation one observes using staggered fermions, see, e.g. Ref. [19].

For the small quark-disconnected contribution and the QED and strong isospin-breaking corrections we currently only use one lattice cutoff $\left(a^{-1}=1.73 \mathrm{GeV}\right)$ and include an appropriate estimate of cutoff effects in the final analysis. 

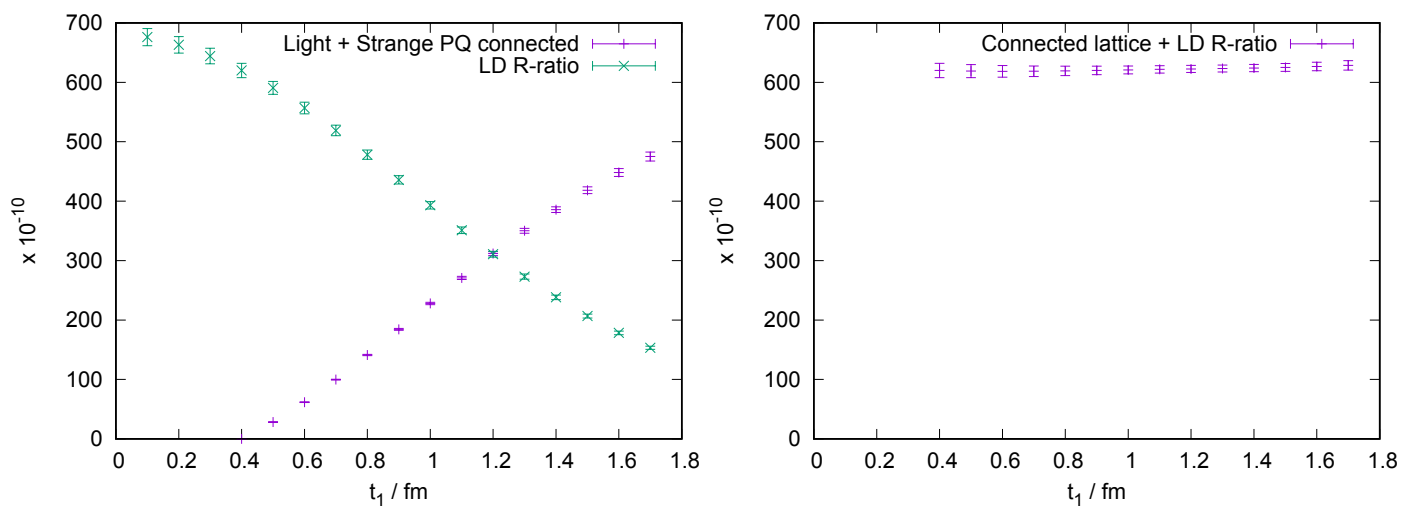

Figure 14. Lattice and R-ratio data contributions seperate (left) and summed (right) as a function of $t_{1}$. We notice that while the individual contributions vary on the order of the total $a_{\mu}$ in this range of $t_{1}$, the sum is constant within its uncertainty. This provides a strong consistency check between the lattice and the R-ratio data.

\subsection{Combined analysis}

As explained above, we take $a_{\mu}^{\mathrm{LD}}$ and $a_{\mu}^{\mathrm{SD}}$ from the R-ratio data and combine it with $a_{\mu}^{\mathrm{W}}$ corresponding to the continuum and infinite-volume limit of the quark-connected, quark-disconnected, and QED and strong isospin-breaking contributions that we have discussed previously. The contributions of up, down, strange, and charm quarks are included.

In Fig. 14 we show $a_{\mu}^{\mathrm{LD}}$ and $a_{\mu}^{\mathrm{W}}$ both individually as well as their sum for fixed $t_{0}=0.4 \mathrm{fm}$ and $\Delta=0.15 \mathrm{fm}$ as a function of $t_{1}$. In Fig. 14 only the isospin symmetric light and strange quarkconnected data is used for the lattice data. In Fig. 15, we show the quantitative effect of additional corrections to the lattice data: i) the addition of scalar QED finite-volume corrections, ii) the addition of quark-disconnected contributions, iii) the addition of strong isospin-breaking diagram $\mathrm{M}$, iv) the addition of QED corrections $\mathrm{S}+\mathrm{V}, \mathrm{v}$ ) the addition of QED correction F, and finally vi) the addition of the quark-connected charm-quark contribution.

We observe that by changing $t_{1}$ from $0.4 \mathrm{fm}$ to $1.7 \mathrm{fm}$ we can vary the fractional individual contributions from a region where the result is given completely by the R-ratio data, to a region where the result is mostly given by the lattice data. In this range the individual contributions therefore change on the order of $a_{\mu}^{\mathrm{HVP} L O}$. We note that the sum of both contributions is constant within errors, which is a strong test of consistency between lattice and R-ratio data. As the lattice data becomes more precise in the future, the total uncertainty can be reduced and longer distance contributions can be calculated from ab-initio lattice computations. In an upcoming paper [2], we also present results with $a_{\mu}^{\mathrm{SD}}$ and $a_{\mu}^{\mathrm{LD}}$ obtained from the lattice directly.

\section{Conclusion}

I have presented the current status of a first-principles calculation at physical pion mass of the quark-connected, quark-disconnected, leading QED and strong isospin-breaking contributions to the leading-order HVP performed by the RBC and UKQCD collaborations.

The light-quark quark-connected contribution is computed with an improved statistical estimator that reduces the statistical noise at same cost compared to the usual $Z_{2}$ wall source method by an order of magnitude. In addition a new Lanczos method using multiple grids [12] further improves the 


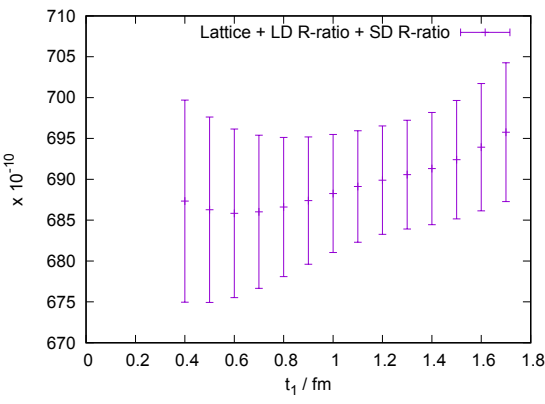

Identical to Fig. 14

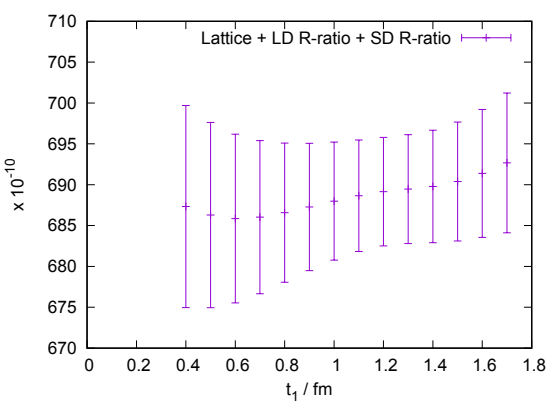

quark-disconnected contribution

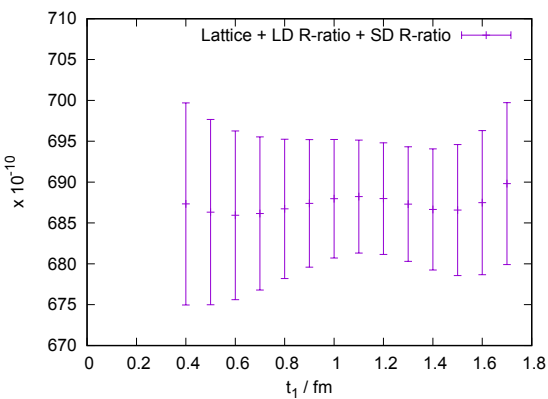

Diagrams S+V

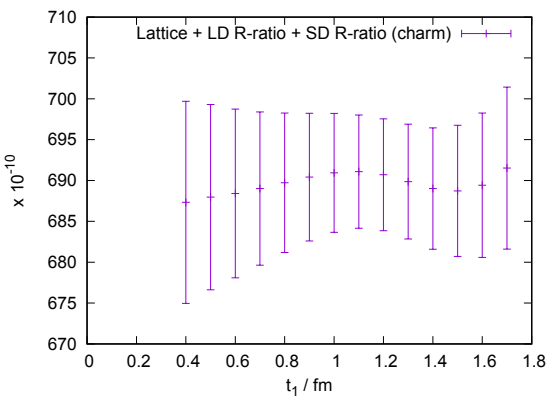

charm quark-connected contribution

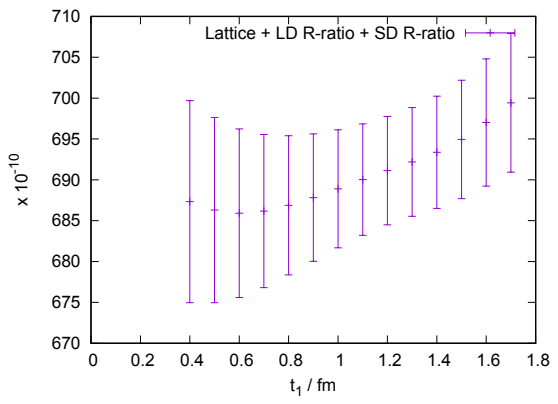

sQED finite-volume correction

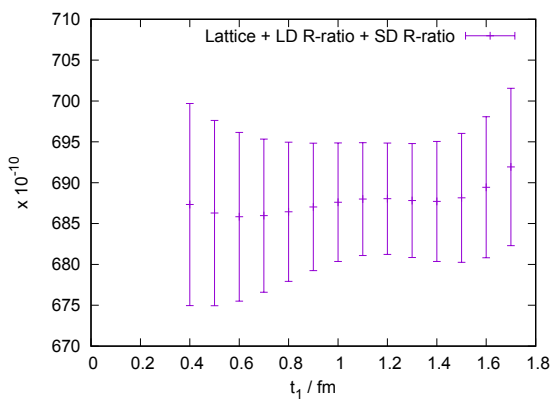

Diagram M

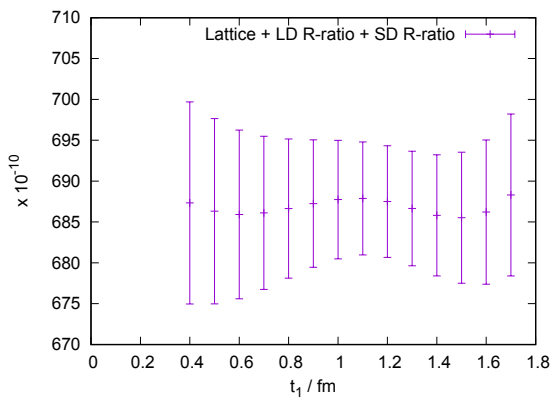

Diagram F

Figure 15. Effect of individual corrections to the isospin-symmetric quark-connected light and strange quark lattice data. Contributions are accumulated from the top left to the bottom right labeling each new contribution. 


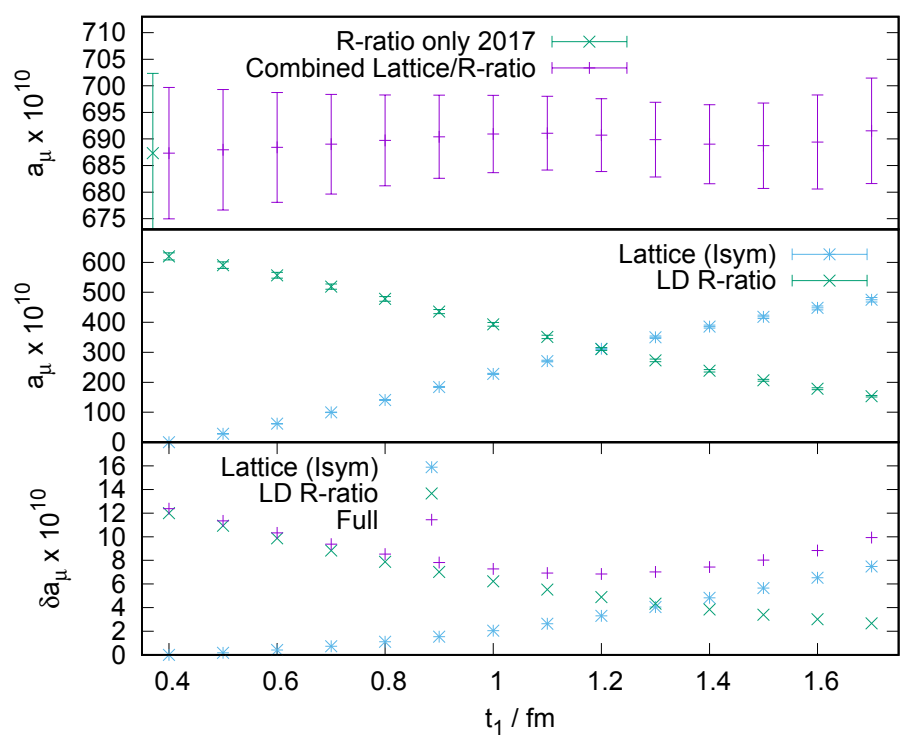

Figure 16. The top panel shows the combined lattice and R-ratio result as a function of $t_{1}$. We also show a pure R-ratio result based on the dataset of [18] ignoring the correlations between different energies. The middle panel separates the size of the lattice and R-ratio contributions and the bottom panel shows the magnitude of individual contributions to the combined uncertainty.

efficiency of this method by reducing the memory cost by an order of magnitude. We combine this new dataset with the strange-quark quark-connected and the quark-disconnected contributions already published in Refs. [13] and [14].

For the QED contributions we used a stochastic position-space sampling technique similar to the one used in our hadronic light-by-light calculation of Ref. [15]. We have re-tuned the quark masses and checked the prediction of the pion mass splitting against the experimental result. The re-tuning of the lattice spacing and the QED corrections to the local current normalization are missing in this presentation but will be published in Ref. [2].

For the charm-quark contribution we use the vector correlator of a heavy quark tuned by matching the heavy-light pseudo-scalar meson mass to the $D$ meson mass.

We have devised a window method to combine and cross-check lattice and R-ratio data. We summarize the results again in Fig. 16. This method allows for further reduction in uncertainty over the already very precise R-ratio results. In the results presented here, correlations between different energies in the R-ratio are not yet taken into account, however, this imperfection will be removed in Ref. [2].

With these limitations we obtained a result with total uncertainty of $\delta a_{\mu}^{\mathrm{HVP} \mathrm{LO}}=6.8 \times 10^{-10}$. A full discussion of systematic uncertainties will be provided in Ref. [2].

We note that the window can eventually be widened to obtain a pure lattice result with adequate precision matching the Fermilab E989 experiment. In the near future, however, the combination of R-ratio data with lattice data has the potential to provide the most precise determination of the HVP. We conclude with showing our preliminary result in context of recent pure lattice and R-ratio determinations in Fig. 17. 


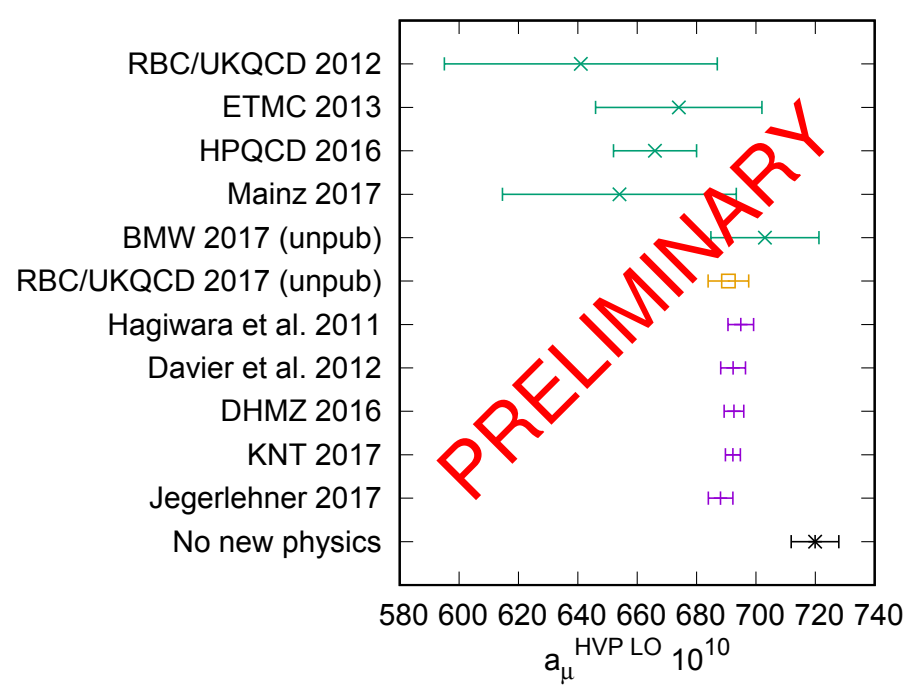

Figure 17. Overview over recent lattice and R-ratio results.

Acknowledgments. The presented work is performed in collaboration with colleagues of the RBC and UKQCD collaborations. We are also indebted to Kim Maltman for a fruitful collaboration. C.L. acknowledges support through a DOE Office of Science Early Career Award and by US DOE Contract DESC0012704(BNL). Computational resources provided by the clusters operated by USQCD at Fermilab and Jefferson Laboratory, by the Dirac facility at the University of Edinburgh, by the KNL system operated by the CSI at Brookhaven National Laboratory, and by the Mira facility at Argonne National Laboratory are gratefully acknowledged. 


\section{References}

[1] T. Blum et al. (RBC, UKQCD), Phys. Rev. D93, 074505 (2016), 1411.7017

[2] C. Lehner et al. (RBC, UKQCD), In preparation

[3] M. Della Morte, A. Francis, V. Gülpers, G. Herdoíza, G. von Hippel, H. Horch, B. Jäger, H.B. Meyer, A. Nyffeler, H. Wittig, JHEP 10, 020 (2017), 1705.01775

[4] D. Bernecker, H.B. Meyer, Eur. Phys. J. A47, 148 (2011), 1107.4388

[5] T. Blum, Phys.Rev.Lett. 91, 052001 (2003), hep-lat/0212018

[6] C. Aubin, T. Blum, P. Chau, M. Golterman, S. Peris, C. Tu, Phys. Rev. D93, 054508 (2016), 1512.07555

[7] T.A. DeGrand, S. Schaefer, Nucl. Phys. Proc. Suppl. 140, 296 (2005), [,296(2004)], hep-lat/0409056

[8] S. Collins, G. Bali, A. Schafer, PoS LAT2007, 141 (2007), 0709. 3217

[9] G.S. Bali, S. Collins, A. Schafer, Comput. Phys. Commun. 181, 1570 (2010), 0910. 3970

[10] T. Blum, T. Izubuchi, E. Shintani, Phys. Rev. D88, 094503 (2013), 1208.4349

[11] E. Shintani, R. Arthur, T. Blum, T. Izubuchi, C. Jung, C. Lehner, Phys. Rev. D91, 114511 (2015), 1402.0244

[12] K. Clark, C. Jung, C. Lehner, poster contributed to Lattice 2017

[13] T. Blum et al. (RBC/UKQCD), JHEP 04, 063 (2016), [Erratum: JHEP05,034(2017)], 1602.01767

[14] T. Blum, P.A. Boyle, T. Izubuchi, L. Jin, A. Jüttner, C. Lehner, K. Maltman, M. Marinkovic, A. Portelli, M. Spraggs, Phys. Rev. Lett. 116, 232002 (2016), 1512.09054

[15] T. Blum, N. Christ, M. Hayakawa, T. Izubuchi, L. Jin, C. Lehner, Phys. Rev. D93, 014503 (2016), 1510.07100

[16] S. Borsanyi et al., Science 347, 1452 (2015), 1406. 4088

[17] P. Boyle, V. Gülpers, J. Harrison, A. Jüttner, C. Lehner, A. Portelli, C.T. Sachrajda, JHEP 09, 153 (2017), 1706.05293

[18] F. Jegerlehner, http://www-com.physik.hu-berlin.de/ fjeger/software.html

[19] B. Chakraborty, C.T.H. Davies, P.G. de Oliviera, J. Koponen, G.P. Lepage, R.S. Van de Water, Phys. Rev. D96, 034516 (2017), 1601.03071 